\title{
Fuzzy Functional and Multivalued Dependencies for Frank's Class of Additive Generators
}

\author{
Sanela Nesimović \\ Faculty of Educational Sciences, University of Sarajevo \\ Skenderija 72, 71000 Sarajevo \\ Bosnia and Herzegovina \\ snesimovic@pf.unsa.ba \\ Dženan Gušić \\ Department of Mathematics, Faculty of Sciences and Mathematics, University of Sarajevo \\ Zmaja od Bosne 33-35, 71000 Sarajevo \\ Bosnia and Herzegovina \\ dzenang@pmf.unsa.ba
}

Received: June 7, 2020. Revised: December 17, 2020. Accepted: January 8, 2021. Published: January 18, 2021.

\begin{abstract}
In this paper we consider all possible dependencies that can be built upon similarity-based fuzzy relations, that is, fuzzy functional and fuzzy multivalued dependencies. Motivated by the fact that the classical obtaining of new dependencies via inference rules may be tedious and uncertain, we replace it by the automated one, where the key role is played by the resolution principle techniques and the fuzzy formulas in place of fuzzy dependencies. We prove that some fuzzy multivalued dependency is actively correct with respect to given fuzzy relation instance if and only if the corresponding fuzzy formula is in line with the attached interpretation. Additionally, we require the tuples of the instance to be conformant (up to some extent) on the leading set of attributes. The equivalence as well as the conclusion are generalized to sets of attributes. The research is conducted by representing the attributes and fuzzy dependencies in the form of fuzzy formulas, and the application of fuzzy implication operators derived from carefully selected Frank's classes of additive generators.
\end{abstract}

Keywords- Fuzzy functional and multivalued dependencies, Frank's class, fuzzy formulas, fuzzy relations.

\section{INTRODUCTION}

BESIDES functional dependencies, multivalued dependencies represent yet another integrity constraint that can be build upon tuples of a database [1].

Simply speaking, a value of an attribute (or values of a set of attributes) are compared to a set of values attached to a set of attributes. More precisely, relation instance $r$ is said to satisfy the multivalued dependency $X \rightarrow \rightarrow Y$ ( $X$ multidetermines $Y$ ) if for each $t_{1}, t_{2} \in r$, the equality $t_{1}[X]=$
$t_{2}[X]$ yields that there is some $t_{3} \in r$, such that $t_{3}[X]=$ $t_{1}[X], t_{3}[Y]=t_{1}[Y]$, and $t_{3}\left[(X \cup Y)^{c}\right]=t_{2}\left[(X \cup Y)^{c}\right]$, with $(X \cup Y)^{c}$ the complement of $X \cup Y$ in the universal set of attributes.

So, multivalued dependencies are formulated in the same way as the remaining constraints in relational databases. As in the case of functional (fuzzy functional) dependencies, the transition from classical to fuzzy case is accomplished in [2]. The approach is adapted in a way to include precise and imprecise dependencies by accepting a $[0,1]$-value designated as a linguistic strength of the dependency. The inference rules are given, and are shown to be consistent, sound and complete.

Generally, not so much is done to describe and formally define fuzzy multivalued dependencies. Besides [2], the authors in [3] argue that any fuzzy multivalued dependency should have a threshold valued $\theta$, such that each time tuples are more similar than $\theta$, the dependency should check whether or not the third tuple exists. The approach [2] simplifies things and removes the threshold value, however.

The authors in [4] apply the theory of possibility distribution and thus show that multivalued dependencies can be expressed in the frame work of particularization. The validity of inference rules for multivalued dependencies in fuzzy relations has also been discussed.

On the other side, in [5], the decision whether a tuple satisfies the multivalued dependency is determined by comparing the compatibility degree with the value of membership attribute. The value $t\left[A_{j}\right]$ of an attribute $A_{j}$ in a tuple $t$ is represented by a normal possibility distribution, i.e.,

$$
t\left[A_{j}\right]=\sum_{u \in D_{j}} \pi_{t\left[A_{j}\right]}(u) / u,
$$




$$
\sup _{u \in D_{j}} \pi_{t\left[A_{j}\right]}(u)=1
$$

where each domain is a crisp set consisting of normal elements and the one denoted as NULL. New, sound and complete inference rules have been described.

The ordinary relational database theory [6] forces the database designer to operate only with a restricted design (assuming all of the information to be known and exact). Several approaches are made in order to include fuzzy data into classical database theory: [7] (fuzzy membership values), [8]-[10] (possibility distributions), and [11]-[13] (similarity relations).

The research conducted here assumes the similarity-based fuzzy relational database approach.

The results derived in [2] are raised to higher level in [14], where several equivalences are discussed and proved. In particular, following concept of "satisfiability" (see, [15]), the authors in [14] represented fuzzy dependencies as fuzzy formulas, and thus enabled resolution principle techniques to be applied in the process of generating new dependencies. The results initially derived for Kleene-Dienes fuzzy implication operator are verified later on in [16] and [17], [18] for families of $f$-generated and $g$-generated implications, respectively (see also, [19] and [20] for Reichenbach and Klir-Yuan implications).

Following reasoning in the move from fuzzy functional to vague functional dependencies [21], a similar transfer is done with fuzzy multivalued (vague multivalued) dependencies in [22]. New definition of vague multivalued dependencies is given, the inference rules are listed, and are proven to be sound. Although various similarity measures were known to be present in literature: $\mathrm{Lu}-\mathrm{Ng}$ [23], Chen [24], Hong-Kim [25], Li-Xu [26], Szmidt-Kacprzyk [27], etc., the author in [22] opted for a general similarity measure assuming it to be reflexive, symmetric, and max-min transitive (Cf. [2]).

Motivated by the fact that the inclusive and the augment rules are sound, the research is continued in [28], so it was proven that complementation, transitivity, replication, coalescence, union, pseudo-transitivity, and mixed transitivity are also sound rules. The completeness of the inference rules is discussed in [29] in the case of two-element vague relation instances, and then in [30] in the case of arbitrary vague relations (see also, [31]-[33]).

We notice that the authors in [34] introduced a new definition of vague multivalued dependencies, called $\alpha$-vague multivalued dependencies. The definition is given on the basis of $\alpha$-equality between tuples, and is shown to be consistent, while the set of the inference rules is proven to be sound and complete.

Here, in view of Lee's work [15], attributes and fuzzy dependencies are considered as fuzzy formulas (with respect to appropriately selected interpretations). Motivated by the fact that the classical obtaining of new fuzzy dependencies (functional and multivalued) is usually very difficult, we replace traditional techniques with resolution ones, which make possible the use of automatization.Thus, we prove that some fuzzy multivalued dependency is actively true with regard to given fuzzy relation instance if and only if the associated fuzzy formula fully agrees with the attached interpretation (requiring additionally the tuples of the instance to be simi- lar enough on the leading attributes set). The equivalence is given by Theorem 1 . In Theorems 2 and 3, we prove that the analogous equivalence can be obtained for sets of attributes by natural transferring from a single attribute to any set of attributes.

The research is accomplished with the use of Frank's classes of additive generators.

\section{PRELIMINARIES}

We assume that the fuzzy logic operators: conjunction and disjunction are given by (see, [35] and [36]) $C(x, y)=$ $\min \{x, y\}$ and $D(x, y)=\max \{x, y\}$, respectively.

Following work of Lee [15], we say that fuzzy formula $f$ is satisfied (violated) by interpretation $I$, if $T(f) \geq 0.5(T(f)$ $\leq 0.5$ ) with respect to $I$. If $T(f) \leq 0.5$ for every $I$, we say that fuzzy formula $f$ is contradictory.

Let $r$ be fuzzy relation instance on scheme $R$, and $U$ be the universal set of attributes. Suppose that $X$ and $Y$ are subsets of $U$, and that $\theta \in[0,1]$ is a number.

We say that fuzzy multivalued dependency $X \rightarrow \stackrel{\theta}{\rightarrow}_{F} Y$ is satisfied by $r$, if for every pair of tuples $t_{1}$ and $t_{2}\left(t_{1}, t_{2} \in r\right)$, there exists a tuple $t_{3} \in r$, such that

$$
\begin{aligned}
\varphi\left(X\left[t_{3}, t_{1}\right]\right) & \geq \min \left\{\theta, \varphi\left(X\left[t_{1}, t_{2}\right]\right)\right\}, \\
\varphi\left(Y\left[t_{3}, t_{1}\right]\right) & \geq \min \left\{\theta, \varphi\left(X\left[t_{1}, t_{2}\right]\right)\right\}, \\
\varphi\left(U \backslash(X \cup Y)\left[t_{3}, t_{2}\right]\right) & \geq \min \left\{\theta, \varphi\left(X\left[t_{1}, t_{2}\right]\right)\right\} .
\end{aligned}
$$

The number $\theta$ is called the linguistic strength of the dependency $X \rightarrow \stackrel{\theta}{\rightarrow}_{F} Y$.

Furthermore, $\varphi\left(X\left[t_{3}, t_{1}\right]\right)$ is used to denote the conformance between tuples $t_{3}$ and $t_{1}$ on set of attributes $X$. Thus, to properly define $\varphi\left(X\left[t_{3}, t_{1}\right]\right)$, one has to define the conformance between tuples on a single attribute.

The conformance of attribute $A_{k}$ (defined on domain $D_{k}$ ) on tuples $t_{i}$ and $t_{j}\left(t_{i}\right.$ and $t_{j}$ belong to some fuzzy relation instance $r)$, is denoted by $\varphi\left(A_{k}\left[t_{i}, t_{j}\right]\right)$, and is given by

$$
\begin{aligned}
& \varphi\left(A_{k}\left[t_{i}, t_{j}\right]\right) \\
= & \min \left\{\min _{x \in d_{i}}\left\{\max _{y \in d_{j}}\{s(x, y)\}\right\},\right. \\
& \left.\min _{x \in d_{j}}\left\{\max _{y \in d_{i}}\{s(x, y)\}\right\}\right\},
\end{aligned}
$$

where $d_{i}$ is the value of the attribute $A_{k}$ on $t_{i}, d_{j}$ is the value of $A_{k}$ on $t_{j}$, and $s(x, y)$ is a similarity relation between values $x$ and $y$.

If $\varphi\left(A_{k}\left[t_{i}, t_{j}\right]\right) \geq \theta$, the tuples $t_{i}$ and $t_{j}$ are said to be conformant on attribute $A_{k}$ with $\theta$.

Consequently, the definition is extended to describe the conformance between tuples on sets of attributes.

In particular, the conformance between tuples $t_{i}$ and $t_{j}$ on attribute set $X$ i denoted by $\varphi\left(X\left[t_{i}, t_{j}\right]\right)$, and is defined by 


$$
\begin{aligned}
& \varphi\left(X\left[t_{i}, t_{j}\right]\right) \\
= & \min \left\{\varphi\left(A\left[t_{i}, t_{j}\right]\right): A \in X\right\} .
\end{aligned}
$$

In order to turn the attributes and fuzzy dependencies into fuzzy formulas, we introduce the concept of valuation (see, [15]).

If $r=\left\{t_{i}, t_{j}\right\}$ is a relation on scheme $R$, and $\theta \in[0,1]$ is a value, we define the valuation (interpretation) $i_{r, \theta}(A)$ of the attribute $A$ by

$$
\begin{cases}>0.5, & \varphi\left(A\left[t_{i}, t_{j}\right]\right) \geq \theta \\ \leq 0.5, & \varphi\left(A_{k}\left[t_{i}, t_{j}\right]\right)<\theta\end{cases}
$$

Turning in this way the attributes into fuzzy formulas, we enable fuzzy dependencies to be treated as fuzzy formulas as well. Thus, we join the fuzzy formula

$$
\begin{aligned}
& \left(A_{1} \wedge \ldots \wedge A_{m}\right) \Rightarrow \\
& \left(\left(B_{1} \wedge \ldots \wedge B_{n}\right) \vee\left(C_{1} \wedge \ldots \wedge C_{p}\right)\right)
\end{aligned}
$$

to fuzzy multivalued dependency $X \rightarrow \stackrel{\theta}{\rightarrow}_{F} Y$, where $X=$ $\left\{A_{1}, \ldots, A_{m}\right\}, Y=\left\{B_{1}, \ldots, B_{n}\right\}$ and

$U \backslash(X \cup Y)=\left\{C_{1}, \ldots, C_{p}\right\}$.

If it happens that $r$ satisfies $X \rightarrow \stackrel{\theta}{\rightarrow}_{F} Y$ in the way that $\varphi\left(X\left[t_{i}, t_{j}\right]\right) \geq \theta$ for $t_{i}, t_{j} \in r$, we say that $X \rightarrow \stackrel{\theta}{\rightarrow}_{F} Y$ is $\theta-$ actively correct with respect to $r$. Clearly, it is the case if and only if $\varphi\left(X\left[t_{1}, t_{2}\right]\right) \geq \theta, \varphi\left(Y\left[t_{1}, t_{2}\right]\right) \geq \theta$ or $\varphi\left(X\left[t_{1}, t_{2}\right]\right)$ $\geq \theta, \varphi\left(U \backslash(X \cup Y)\left[t_{1}, t_{2}\right]\right) \geq \theta$ when $r=\left\{t_{1}, t_{2}\right\}$.

In this research we consider the Frank's class of additive generators defined by

$$
f^{a}(x)=-\ln \left(\frac{a^{x}-1}{a-1}\right),
$$

where $a>0, a \neq 1$.

Note that $f^{a}(0)=\infty$, and that

$$
\left(f^{a}(x)\right)^{-1}=\log _{a}\left(1+(a-1) e^{-x}\right) .
$$

Therefore, the fuzzy implication determined by $f$ states that

$$
I_{f^{a}}(x, y)=\log _{a}\left(1+(a-1)^{1-x}\left(a^{y}-1\right)^{x}\right)
$$

for $x, y \in[0,1]$.

Furthermore, we are interested in Frank's class of additive generators introduced by

$$
g^{b}(x)=-\ln \left(\frac{b^{1-x}-1}{b-1}\right),
$$

where $b>0, b \neq 1$.

In this case, $g^{b}(1)=\infty$, and

$$
\left(g^{b}(x)\right)^{-1}=1-\log _{b}\left(1+(b-1) e^{-x}\right) .
$$

The corresponding fuzzy implication states that

$$
I_{g^{b}}(x, y)=1-\log _{b}\left(1+(b-1)^{\frac{x-1}{x}}\left(b^{1-y}-1\right)^{\frac{1}{x}}\right)
$$

for $x, y \in[0,1]$.

Note that $f^{a}(1)=0$. Moreover,

$$
\begin{aligned}
\frac{d}{d x} f^{a}(x) & =-\frac{d}{d x} \ln \left(a^{x}-1\right) \\
& =-\frac{1}{a^{x}-1} a^{x} \ln a<0,
\end{aligned}
$$

so $f^{a}(x)$ is a strictly decreasing function $\left(f^{a}:[0,1] \rightarrow\right.$ $[0,+\infty])$, which is also continuous.

On the other side, $g^{b}(0)=0$. Furthermore,

$$
\begin{aligned}
\frac{d}{d x} g^{b}(x) & =-\frac{d}{d x} \ln \left(b^{1-x}-1\right) \\
& =\frac{1}{b^{1-x}-1} b^{1-x} \log b>0
\end{aligned}
$$

so the function $g^{b}(x)$ is strictly increasing $\left(g^{b}:[0,1] \rightarrow\right.$ $[0,+\infty])$, and is also continuous one.

\section{RESULTS}

Theorem 1. Let $R$ be a relation scheme, and $X=$ $\left\{A_{1}, \ldots, A_{m}\right\}, Y=\left\{B_{1}, \ldots, B_{n}\right\}, Z=\left\{C_{1}, \ldots, C_{p}\right\}$ be subsets of the set of all attributes. If $r$ is a two-element relation over $R$, and $0 \leq \theta \leq 1$ is a number, then, the fuzzy multivalued dependency $X \rightarrow \stackrel{\theta}{\rightarrow}_{F} Y$ is $\theta$-actively correct with respect to $r$ if and only if the tuples of $r$ are conformant with $\theta$ on $X$, and

$$
\begin{aligned}
\mathcal{H}: & \left(A_{1} \wedge \ldots \wedge A_{m}\right) \Rightarrow \\
& \left(\left(B_{1} \wedge \ldots \wedge B_{n}\right) \vee\left(C_{1} \wedge \ldots \wedge C_{p}\right)\right)
\end{aligned}
$$

is valid under the attached valuation $i_{r, \theta}$. Here, we assume that the set of all attributes is given as the union of the sets $X, Y$ and $Z$.

Proof. $(\Rightarrow)$ First, we deal with the implication $I_{f^{a}}(x, y)$.

Since $\varphi\left(X\left[t_{1}, t_{2}\right]\right) \geq \theta, \varphi\left(Y\left[t_{1}, t_{2}\right]\right) \geq \theta$ or $\varphi\left(X\left[t_{1}, t_{2}\right]\right) \geq \theta, \varphi\left(Z\left[t_{1}, t_{2}\right]\right) \geq \theta$, we may assume without loss of generality that the first scenario is true.

We have $X=\left\{A_{1}, \ldots, A_{m}\right\}, Y=\left\{B_{1}, \ldots, B_{n}\right\}, Z=$ $\left\{C_{1}, \ldots, C_{p}\right\}$.

Thus,

$$
\begin{aligned}
\theta \leq & \varphi\left(X\left[t_{1}, t_{2}\right]\right) \\
& \min \left\{\varphi\left(A_{1}\left[t_{1}, t_{2}\right]\right), \ldots, \varphi\left(A_{m}\left[t_{1}, t_{2}\right]\right)\right\},
\end{aligned}
$$

so $\varphi\left(A_{1}\left[t_{1}, t_{2}\right]\right) \geq \theta, \ldots, \varphi\left(A_{m}\left[t_{1}, t_{2}\right]\right) \geq \theta$, i.e. $\varphi\left(A_{k}\left[t_{1}, t_{2}\right]\right) \geq \theta$ for all $k \in\{1,2, \ldots, m\}$. 
It follows that $i_{r, \theta}\left(A_{k}\right)>\frac{1}{2}$ for all $k \in$ $\{1,2, \ldots, m\}$.

Hence,

$$
\begin{aligned}
& i_{r, \theta}\left(A_{1} \wedge \ldots \wedge A_{m}\right) \\
= & \min \left\{i_{r, \theta}\left(A_{1}\right), \ldots, i_{r, \theta}\left(A_{m}\right)\right\}>\frac{1}{2} .
\end{aligned}
$$

Reasoning in the same way, we obtain that

$$
i_{r, \theta}\left(B_{1} \wedge \ldots \wedge B_{n}\right)>\frac{1}{2}
$$

We deduce,

$$
\begin{aligned}
& i_{r, \theta}(\mathcal{H}) \\
= & i_{r, \theta}\left(\left(A_{1} \wedge \ldots \wedge A_{m}\right) \Rightarrow\right. \\
& \left.\left(\left(B_{1} \wedge \ldots \wedge B_{n}\right) \vee\left(C_{1} \wedge \ldots \wedge C_{p}\right)\right)\right) \\
= & \log _{a}\left(1+(a-1)^{1-i_{r, \theta}\left(A_{1} \wedge \ldots \wedge A_{m}\right)} \times\right. \\
\times & \left(a^{i_{r, \theta}\left(\left(B_{1} \wedge \ldots \wedge B_{n}\right) \vee\left(C_{1} \wedge \ldots \wedge C_{p}\right)\right)}-1\right) \\
i_{r, \theta}\left(A_{1} \wedge \ldots \wedge A_{m}\right) & \\
= & \log _{a}\left(1+(a-1)^{1-i_{r, \theta}\left(A_{1} \wedge \ldots \wedge A_{m}\right)} \times\right. \\
\times & \left(a^{\max \left\{i_{r, \theta}\left(B_{1} \wedge \ldots \wedge B_{n}\right), i_{r, \theta}\left(C_{1} \wedge \ldots \wedge C_{p}\right)\right\}}-1\right) \\
i_{r, \theta}\left(A_{1} \wedge \ldots \wedge A_{m}\right) & \\
= & \log _{a}\left(1+(a-1)^{1-A}\left(a^{B}-1\right)^{A}\right) .
\end{aligned}
$$

Having in mind that

$$
\begin{aligned}
& i_{r, \theta}\left(A_{1} \wedge \ldots \wedge A_{m}\right)>\frac{1}{2}, \\
& i_{r, \theta}\left(B_{1} \wedge \ldots \wedge B_{n}\right)>\frac{1}{2},
\end{aligned}
$$

we immediately have that $A>\frac{1}{2}$.

If

$$
\begin{aligned}
& \max \left\{i_{r, \theta}\left(B_{1} \wedge \ldots \wedge B_{n}\right), i_{r, \theta}\left(C_{1} \wedge \ldots \wedge C_{p}\right)\right\} \\
= & i_{r, \theta}\left(B_{1} \wedge \ldots \wedge B_{n}\right)>\frac{1}{2}
\end{aligned}
$$

then $B>\frac{1}{2}$.

Also, if

$$
\begin{aligned}
& \max \left\{i_{r, \theta}\left(B_{1} \wedge \ldots \wedge B_{n}\right), i_{r, \theta}\left(C_{1} \wedge \ldots \wedge C_{p}\right)\right\} \\
= & i_{r, \theta}\left(C_{1} \wedge \ldots \wedge C_{p}\right) \\
\geq & i_{r, \theta}\left(B_{1} \wedge \ldots \wedge B_{n}\right)>\frac{1}{2},
\end{aligned}
$$

then $B>\frac{1}{2}$.
In any case, $A, B>\frac{1}{2}$.

Suppose that $B=1$. We have,

$$
\begin{aligned}
& i_{r, \theta}(\mathcal{H}) \\
= & \log _{a}\left(1+(a-1)^{1-A}(a-1)^{A}\right) \\
= & \log _{a}(1+a-1)=1>\frac{1}{2} .
\end{aligned}
$$

Now, let $B<1$.

Suppose that $i_{r, \theta}(\mathcal{H}) \leq \frac{1}{2}$.

We have,

$$
\begin{aligned}
\frac{1}{2} & \geq \log _{a}\left(1+(a-1)^{1-A}\left(a^{B}-1\right)^{A}\right) \\
& =\log _{a}\left(a+(a-1)\left(\frac{a^{B}-1}{a-1}\right)^{A}\right) \\
& =\log _{a}\left(a+(a-1) e^{-A\left(-\ln \left(\frac{a^{B}-1}{a-1}\right)\right)}\right) \\
& =\log _{a}\left(1+(a-1) e^{-A f^{a}(B)}\right) \\
& =\left(f^{a}\left(A f^{a}(B)\right)\right)^{-1} .
\end{aligned}
$$

Since $f^{a}$ is strictly decreasing function, we have that $f^{a}\left(\frac{1}{2}\right) \leq A f^{a}(B)$. The fact that $B>\frac{1}{2}$ implies that

$$
A \geq \frac{f^{a}\left(\frac{1}{2}\right)}{f^{a}(B)}>1,
$$

which is a contradiction.

Consequently, $i_{r, \theta}(\mathcal{H})>\frac{1}{2}$.

Now, consider the case of the implication $I_{g^{b}}(x, y)$.

As in the previous case, we have that

$$
\begin{aligned}
i_{r, \theta}\left(A_{1} \wedge \ldots \wedge A_{m}\right)>\frac{1}{2}, \\
i_{r, \theta}\left(B_{1} \wedge \ldots \wedge B_{n}\right)>\frac{1}{2} .
\end{aligned}
$$

We deduce,

$$
\begin{aligned}
& i_{r, \theta}(\mathcal{H}) \\
= & i_{r, \theta}\left(\left(A_{1} \wedge \ldots \wedge A_{m}\right) \Rightarrow\right. \\
& \left.\left(\left(B_{1} \wedge \ldots \wedge B_{n}\right) \vee\left(C_{1} \wedge \ldots \wedge C_{p}\right)\right)\right) \\
= & 1-\log _{b}\left(1+(b-1)^{\frac{i_{r, \theta}\left(A_{1} \wedge \ldots \wedge A_{m}\right)-1}{i_{r, \theta}\left(A_{1} \wedge \ldots \wedge A_{m}\right)}} \times\right. \\
& \left(b^{1-i_{r, \theta}\left(\left(B_{1} \wedge \ldots \wedge B_{n}\right) \vee\left(C_{1} \wedge \ldots \wedge C_{p}\right)\right)}-1\right) \\
& \left.\frac{1}{i_{r, \theta}\left(A_{1} \wedge \ldots \wedge A_{m}\right)}\right) \\
= & 1-\log _{b}\left(1+(b-1)^{\frac{i_{r, \theta}\left(A_{1} \wedge \ldots \wedge A_{m}\right)-1}{i_{r, \theta}\left(A_{1} \wedge \ldots \wedge A_{m}\right)}} \times\right. \\
& \left(b^{1-\max \left\{i_{r, \theta}\left(B_{1} \wedge \ldots \wedge B_{n}\right), i_{r, \theta}\left(C_{1} \wedge \ldots \wedge C_{p}\right)\right\}}-1\right)
\end{aligned}
$$




$$
\begin{aligned}
& \left.\frac{1}{i_{r, \theta}\left(A_{1} \wedge \ldots \wedge A_{m}\right)}\right) \\
= & 1-\log _{b}\left(1+(b-1)^{\frac{A-1}{A}}\left(b^{1-B}-1\right)^{\frac{1}{A}}\right) .
\end{aligned}
$$

Note that $A>\frac{1}{2}, B>\frac{1}{2}$.

We may write,

$$
\begin{aligned}
& i_{r, \theta}(\mathcal{H}) \\
= & 1-\log _{b}\left(1+(b-1)^{1-\frac{1}{A}}\left(b^{1-B}-1\right)^{\frac{1}{A}}\right) \\
= & 1-\log _{b}\left(1+(b-1)\left(\frac{b^{1-B}-1}{b-1}\right)^{\frac{1}{A}}\right) \\
= & 1-\log _{b}\left(1+(b-1) e^{-\frac{1}{A}\left(-\ln \left(\frac{b^{1-B}-1}{b-1}\right)\right)}\right) \\
= & 1-\log _{b}\left(1+(b-1) e^{-\frac{1}{A} g^{b}(B)}\right) \\
= & \left(g^{b}\left(\frac{1}{A} g^{b}(B)\right)\right)^{-1} .
\end{aligned}
$$

Since $A \leq 1, B>\frac{1}{2}$, and $g^{b}$ is strictly increasing function, we have that

$$
A g^{b}\left(\frac{1}{2}\right) \leq 1 \cdot g^{b}\left(\frac{1}{2}\right)<g^{b}(B)
$$

i.e.,

$$
\frac{1}{A} g^{b}(A)>g^{b}\left(\frac{1}{2}\right)
$$

It immediately follow sthat

$$
\begin{aligned}
i_{r, \theta}(\mathcal{H}) & =\left(g^{b}\left(\frac{1}{A} g^{b}(B)\right)\right)^{-1} \\
& >\left(g^{b}\left(\frac{1}{A} g^{b}\left(\frac{1}{2}\right)\right)\right)^{-1}=\frac{1}{2} .
\end{aligned}
$$

$(\Leftarrow)$ First, consider the case $I_{f^{a}}(x, y)$.

As in the previous direction, we find that

$$
\begin{aligned}
\frac{1}{2} & <i_{r, \theta}(\mathcal{H}) \\
& =\log _{a}\left(1+(a-1)^{1-A}\left(a^{B}-1\right)^{A}\right),
\end{aligned}
$$

where

$$
\begin{aligned}
A= & i_{r, \theta}\left(A_{1} \wedge \ldots \wedge A_{m}\right), \\
B= & \max \left\{i_{r, \theta}\left(B_{1} \wedge \ldots \wedge B_{n}\right),\right. \\
& \left.i_{r, \theta}\left(C_{1} \wedge \ldots \wedge C_{p}\right)\right\} .
\end{aligned}
$$

The assumption $\varphi\left(X\left[t_{1}, t_{2}\right]\right) \geq \theta$ yields that $\varphi\left(A_{k}\left[t_{1}, t_{2}\right]\right) \geq \theta$ for all $k \in\{1,2, \ldots, m\}$.

Hence, $i_{r, \theta}\left(A_{k}\right)>\frac{1}{2}$ for all $k \in\{1,2, \ldots, m\}$, so

$$
\begin{aligned}
A & =i_{r, \theta}\left(A_{1} \wedge \ldots \wedge A_{m}\right) \\
& =\min \left\{i_{r, \theta}\left(A_{1}\right), \ldots, i_{r, \theta}\left(A_{m}\right)\right\}>\frac{1}{2} .
\end{aligned}
$$

In order to complete the proof, it will be enough to prove that $B>\frac{1}{2}$.

Actually, if $B>\frac{1}{2}$, then

$$
i_{r, \theta}\left(B_{1} \wedge \ldots \wedge B_{n}\right)>\frac{1}{2}
$$

or

$$
i_{r, \theta}\left(C_{1} \wedge \ldots \wedge C_{p}\right)>\frac{1}{2}
$$

As we already concluded, the equivalence: $\varphi\left(X\left[t_{1}, t_{2}\right]\right) \geq \theta$ if and only if $i_{r, \theta}\left(A_{1} \wedge \ldots \wedge A_{m}\right)>\frac{1}{2}$ is satisfied.

Therefore, the assumption $B>\frac{1}{2}$ would imply that $\varphi\left(Y\left[t_{1}, t_{2}\right]\right) \geq \theta$ or $\varphi\left(Z\left[t_{1}, t_{2}\right]\right) \geq \theta$, and the proof would be over.

Now, we prove that $B>\frac{1}{2}$.

By our assumption,

$$
\begin{aligned}
\frac{1}{2} & <\log _{a}\left(1+(a-1)^{1-A}\left(a^{B}-1\right)^{A}\right) \\
& =\left(f^{a}\left(A f^{a}(B)\right)\right)^{-1} .
\end{aligned}
$$

Suppose that $i_{r, \theta}(\mathcal{H})=1$.

We obtain, $A f^{a}(B)=0$, i.e., $f^{a}(B)=0$, that is, $B=1$ $>\frac{1}{2}$.

Now, suppose that $i_{r, \theta}(\mathcal{H})<1$.

Since $f^{a}$ is strictly decreasing function, we have that $A f^{a}(B)>0$. Consequently, $f^{a}(B)>0$.

The fact that $i_{r, \theta}(\mathcal{H})>\frac{1}{2}$ implies (as in the previous direction), that $A f^{a}(B)<f^{a}\left(\frac{1}{2}\right)$.

Suppose contrary, that $B \leq \frac{1}{2}$.

The scenario $B=\frac{1}{2}$ implies that $A<1$.

We know that $A>\frac{1}{2}$, so the possibility $A=1$ is not excluded to occur. This means that the statement $A<1$ does not have to be fulfilled.

If we assume that $B<\frac{1}{2}$, then

$$
A<\frac{f^{a}\left(\frac{1}{2}\right)}{f^{a}(B)}<\frac{f^{a}\left(\frac{1}{2}\right)}{f^{a}\left(\frac{1}{2}\right)}=1 .
$$

Once again, the fact that $A>\frac{1}{2}$ does not imply that $A<$ $\frac{f^{a}\left(\frac{1}{2}\right)}{f^{a}(B)}$ must be fulfilled.

Namely, $A$ can occur as a value larger than $\frac{f^{a}\left(\frac{1}{2}\right)}{f^{a}(B)}$.

Hence, $B>\frac{1}{2}$.

As we already noted, this completes the proof for $I_{f^{a}}(x, y)$.

Consider the case $I_{g^{b}}(x, y)$.

Reasoning in the same way as in the previous direction, we end up with 


$$
\begin{aligned}
\frac{1}{2} & <i_{r, \theta}(\mathcal{H}) \\
& =1-\log _{b}\left(1+(b-1)^{\frac{A-1}{A}}\left(b^{1-B}-1\right)^{\frac{1}{A}}\right),
\end{aligned}
$$

where

$$
\begin{aligned}
A= & i_{r, \theta}\left(A_{1} \wedge \ldots \wedge A_{m}\right), \\
B= & \max \left\{i_{r, \theta}\left(B_{1} \wedge \ldots \wedge B_{n}\right),\right. \\
& \left.i_{r, \theta}\left(C_{1} \wedge \ldots \wedge C_{p}\right)\right\} .
\end{aligned}
$$

As above, $\varphi\left(X\left[t_{1}, t_{2}\right]\right) \geq \theta$ if and only if $i_{r, \theta}\left(A_{1} \wedge \ldots \wedge A_{m}\right)>\frac{1}{2}$.

Thus, $A>\frac{1}{2}$.

As in the case of the implication $I_{f^{a}}(x, y)$, we shall prove that $B>\frac{1}{2}$.

By the assumption, we have that

$$
\begin{aligned}
\frac{1}{2} & <1-\log _{b}\left(1+(b-1)^{\frac{A-1}{A}}\left(b^{1-B}-1\right)^{\frac{1}{A}}\right) \\
& =\left(g^{b}\left(\frac{1}{A} g^{b}(B)\right)\right)^{-1} .
\end{aligned}
$$

The function $g^{b}$ is strictly increasing, so

$$
g^{b}\left(\frac{1}{2}\right)<\frac{1}{A} g^{b}(B) .
$$

It follows that $A<\frac{g^{b}(B)}{g^{b}\left(\frac{1}{2}\right)}$.

Note that $A>\frac{1}{2}$. This implies that the quotient $\frac{g^{b}(B)}{g^{b}\left(\frac{1}{2}\right)}$ must be larger than $A$ for any $A>\frac{1}{2}$.

Since $\frac{1}{2} \leq A \leq 1$, this implies that $\frac{g^{b}(B)}{g^{b}\left(\frac{1}{2}\right)}>1$.

In other words, $B>\frac{1}{2}$.

Following lines of the proof for $I_{f^{a}}(x, y)$, we complete the proof.

The main results of the research are given by the following two theorems.

Theorem 2. Let $R$ be a relation scheme. Denote by $C$ a set of fuzzy functional and fuzzy multivalued dependencies on $R$, and by c some particular fuzzy functional (fuzzy multivalued) dependency on $R$. If dependency c follows from the set $C$ in the world of ordinary fuzzy relations, then the fuzzy formula corresponding to $c$ is valid whenever all of fuzzy formulas (corresponding to $C$ ) are valid.

Proof. For the sake of clarity, we denote by $c^{\prime}$ resp. $C^{\prime}$ the formula resp. the set of formulas corresponding to $c$ resp. $C$.

Since we consider two implications $I_{f^{a}}(x, y)$ and $I_{g^{a}}(x, y)$ in this research, we shall prove the claim for $I_{f^{a}}(x, y)$.

By the contra-position law, it is enough to prove that the negated claim yields the negated assumption.

Suppose that the negated claim is fulfilled.
This means that there is some $r=\left\{t_{1}, t_{2}\right\}$, and there is some $\beta \in[0,1]$, such that $i_{r, \beta}(\mathcal{K})>\frac{1}{2}$ for all $\mathcal{K} \in C^{\prime}$, and $i_{r, \beta}\left(c^{\prime}\right) \leq \frac{1}{2}$.

First we prove that the set of those $A$ 's, for which $i_{r, \beta}(A)$ $>\frac{1}{2}$ is non-empty set, and is not whole $U$, where $U$ is the universal set of attributes.

Assume contrary, that $i_{r, \beta}(A) \leq \frac{1}{2}$ for all $A \in U$.

In the case when $c^{\prime}$ corresponds to fuzzy functional dependency $X \stackrel{\theta}{\rightarrow}_{F} Y$, we obtain

$$
\begin{aligned}
\frac{1}{2} \geq & i_{r, \beta}\left(c^{\prime}\right) \\
= & i_{r, \beta}\left(\left(A_{1} \wedge \ldots \wedge A_{m}\right) \Rightarrow\right. \\
& \left.\left(B_{1} \wedge \ldots \wedge B_{n}\right)\right) \\
= & \log _{a}\left(1+(a-1)^{1-i_{r, \beta}\left(A_{1} \wedge \ldots \wedge A_{m}\right)} \times\right. \\
& \left.\times\left(a^{i_{r, \beta}\left(B_{1} \wedge \ldots \wedge B_{n}\right)}-1\right)^{i_{r, \beta}\left(A_{1} \wedge \ldots \wedge A_{m}\right)}\right) \\
= & \log _{a}\left(1+(a-1)^{1-A}\left(a^{B}-1\right)^{A}\right) \\
= & \left(f^{a}\left(A\left(f^{a}(B)\right)\right)\right)^{-1} .
\end{aligned}
$$

Hence, $f^{a}\left(\frac{1}{2}\right) \leq A f^{a}(B)$.

In the case $f^{a}(B)=0$, it follows that $B=1$.

Therefore,

$$
\begin{aligned}
1 & =i_{r, \beta}\left(B_{1} \wedge \ldots \wedge B_{n}\right) \\
& =\min \left\{i_{r, \beta}\left(B_{1}\right), \ldots, i_{r, \beta}\left(B_{n}\right)\right\},
\end{aligned}
$$

so, $i_{r, \beta}\left(B_{k}\right)=1$ for all $k \in\{1,2, \ldots, n\}$.

This means that for at least one $A \in U$, the inequality $i_{r, \beta}(A)>\frac{1}{2}$ holds true.

This is the contradiction.

Consequently, the assumption $f^{a}(B)=0$ leads to contradiction, hence $f^{a}(B)>0$.

We obtain,

$$
A \geq \frac{f^{a}\left(\frac{1}{2}\right)}{f^{a}(B)} .
$$

Note that the assumption $i_{r, \beta}(A) \leq \frac{1}{2}$ for all $A \in U$ implies that

$$
\begin{aligned}
B & =i_{r, \beta}\left(B_{1} \wedge \ldots \wedge B_{n}\right) \\
& =\min \left\{i_{r, \beta}\left(B_{1}\right), \ldots, i_{r, \beta}\left(B_{n}\right)\right\} \leq \frac{1}{2} .
\end{aligned}
$$

Thus,

$$
f^{a}(B) \geq f^{a}\left(\frac{1}{2}\right) .
$$

Taking into account the relations (1) and (2), we conclude that $A$ must be equal to 1 .

Now, reasoning as in the case $B=1$, we conclude that for at least one $A \in U$, the inequality $i_{r, \beta}(A)>\frac{1}{2}$ is satisfied.

In other words, a contradiction. 
Since any scenario, either $f^{a}(B)=0$ or $f^{a}(B)>0$ lead to contradiction, it must be $i_{r, \beta}(A)>\frac{1}{2}$ for at least one $A \in$ $U$.

Now, once again, suppose contrary, that $i_{r, \beta}(A)>\frac{1}{2}$ for all $A \in U$.

The fact that

$$
\frac{1}{2} \geq i_{r \beta}\left(c^{\prime}\right)=\left(f^{a}\left(A\left(f^{a}(B)\right)\right)\right)^{-1}
$$

yields again that $f^{a}\left(\frac{1}{2}\right) \leq A f^{a}(B)$

In the case $f^{a}(B)=0$, we obtain $f^{a}\left(\frac{1}{2}\right) \leq 0$, which is a contradiction, since $f^{a}\left(\frac{1}{2}\right)>0$.

Thus, $f^{a}(B)>0$.

The assumption $i_{r, \beta}(A)>\frac{1}{2}$ for all $A \in U$, gives that

$$
B=\min \left\{i_{r, \beta}\left(B_{1}\right), \ldots, i_{r \beta}\left(B_{n}\right)\right\}>\frac{1}{2} .
$$

We immediately have that

$$
1<\frac{f^{a}\left(\frac{1}{2}\right)}{f^{a}(B)} .
$$

Since $f^{a}\left(\frac{1}{2}\right) \leq A f^{a}(B)$, we must have that $A>1$.

This is not true, however.

The assumption $i_{r, \beta}(A)>\frac{1}{2}$ for all $A \in U$ also leads to contradiction.

We conclude, the set of those $A \in U$, for which $i_{r, \beta}(A)$ $>\frac{1}{2}$, is non-empty, and is not equal to $U$.

If $c^{\prime}$ corresponds to fuzzy multivalued dependency $X$ $\rightarrow \stackrel{\theta}{\rightarrow}_{F} Y$, we argue similarly.

In short, if we assume that $i_{r, \beta}(A) \leq \frac{1}{2}$ for all $A \in U$, we obtain

$$
\begin{aligned}
\frac{1}{2} \geq & i_{r, \beta}\left(c^{\prime}\right) \\
= & i_{r, \beta}\left(\left(A_{1} \wedge \ldots \wedge A_{m}\right) \Rightarrow\right. \\
& \left.\left(\left(B_{1} \wedge \ldots \wedge B_{n}\right) \vee\left(C_{1} \wedge \ldots \wedge C_{p}\right)\right)\right) \\
= & \log _{a}\left(1+(a-1)^{1-i_{r, \beta}\left(A_{1} \wedge \ldots \wedge A_{m}\right)} \times\right. \\
& \times\left(a^{i_{r, \beta}\left(\left(B_{1} \wedge \ldots \wedge B_{n}\right) \vee\left(C_{1} \wedge \ldots \wedge C_{p}\right)\right)}-1\right) \\
& \left.i_{r, \beta}\left(A_{1} \wedge \ldots \wedge A_{m}\right)\right) \\
= & \log _{a}\left(1+(a-1)^{1-i_{r, \beta}\left(A_{1} \wedge \ldots \wedge A_{m}\right)} \times\right. \\
& \times\left(a^{\max \left\{i_{r, \beta}\left(B_{1} \wedge \ldots \wedge B_{n}\right), i_{r, \beta}\left(C_{1} \wedge \ldots \wedge C_{p}\right)\right\}}-1\right) \\
& \left.i_{r, \beta}\left(A_{1} \wedge \ldots \wedge A_{m}\right)\right) \\
= & \log _{a}\left(1+(a-1)^{1-A}\left(a^{B}-1\right)^{A}\right) \\
= & \left(f^{a}\left(A\left(f^{a}(B)\right)\right)\right)^{-1} .
\end{aligned}
$$

As in the previous case $f^{a}\left(\frac{1}{2}\right) \leq A f^{a}(B)$. For $f^{a}(B)=0$, we have that $B=1$.
Hence,

$$
\begin{aligned}
& 1=\max \left\{i_{r, \beta}\left(B_{1} \wedge \ldots \wedge B_{n}\right),\right. \\
& \left.i_{r, \beta}\left(C_{1} \wedge \ldots \wedge C_{p}\right)\right\}
\end{aligned}
$$

i.e.,

$$
\begin{aligned}
& i_{r, \beta}\left(B_{1} \wedge \ldots \wedge B_{n}\right)=1 \\
& i_{r, \beta}\left(C_{1} \wedge \ldots \wedge C_{p}\right)=1
\end{aligned}
$$

In each case we find that there exists at least one $A \in U$, with $i_{r, \beta}(A)>\frac{1}{2}$.

This is the contradiction.

In other words, it must be $f^{a}(B)>0$.

So, (1) remains valid.

The assumption $i_{r, \beta}(A) \leq \frac{1}{2}$ for all $A \in U$ implies as before that

$$
\begin{gathered}
B=\max \left\{i_{r, \beta}\left(B_{1} \wedge \ldots \wedge B_{n}\right),\right. \\
\left.i_{r, \beta}\left(C_{1} \wedge \ldots \wedge C_{p}\right)\right\} \leq \frac{1}{2} .
\end{gathered}
$$

Thus, (2) is also valid.

The relations (1) and (2), however, yield that $A=1$.

As before, this means that must be some $A \in U$, with $i_{r, \beta}(A)>\frac{1}{2}$.

The case $i_{r, \beta}(A)>\frac{1}{2}$ for all $A \in U$ is discussed similarly.

Namely, once again we have that $f^{a}\left(\frac{1}{2}\right) \leq A f^{a}(B)$

If $f^{a}(B)=0$, then $f^{a}\left(\frac{1}{2}\right) \leq 0$, i.e., a contradiction.

If $f^{a}(B)>0$, then the assumption $i_{r, \beta}(A)>\frac{1}{2}$ for all $A$ $\in U$ gives that

$$
\begin{aligned}
B & =\max \left\{i_{r, \beta}\left(B_{1} \wedge \ldots \wedge B_{n}\right),\right. \\
& \left.i_{r, \beta}\left(C_{1} \wedge \ldots \wedge C_{p}\right)\right\} \\
= & \max \left\{\min \left\{i_{r, \beta}\left(B_{1}\right), \ldots, i_{r, \beta}\left(B_{n}\right)\right\},\right. \\
& \left.\min \left\{i_{r, \beta}\left(C_{1}\right), \ldots, i_{r, \beta}\left(C_{p}\right)\right\}\right\}>\frac{1}{2} .
\end{aligned}
$$

So $\sqrt{3}$ is valid, and the fact that $f^{a}\left(\frac{1}{2}\right) \leq$ $A f^{a}(B)$ implies that $A>1$.

This is a contradiction.

Thus, as in the functional case, now, in the multivalued case, the set of those $A \in U$, for which $i_{r, \beta}(A)>\frac{1}{2}$, is nonempty, and is not equal to $U$.

Denote such set of the elements $A \in U$ by $E$.

Using the set $E$, we are in position to define tuples $t^{\prime}$ and $t^{\prime \prime}$ that are conformant on the attributes in $E$.

Denote by $r^{\prime}$ the instance determined by $t^{\prime}$ and $t^{\prime \prime}$.

In order to prove that the negated assumption of theorem is true, it is enough to prove that there is a two-element fuzzy relation instance satisfying $C$, and violating $c$.

We shall prove that $r^{\prime}$ satisfies these conditions.

By the negated claim of theorem, 


$$
\begin{aligned}
\frac{1}{2}< & i_{r, \beta}(\mathcal{K}) \\
= & i_{r, \beta}\left(\left(A_{1} \wedge \ldots \wedge A_{m}\right) \Rightarrow\right. \\
& \left.\left(B_{1} \wedge \ldots \wedge B_{n}\right)\right) \\
= & \log _{a}\left(1+(a-1)^{1-A}\left(a^{B}-1\right)^{A}\right) \\
= & \left(f^{a}\left(A\left(f^{a}(B)\right)\right)\right)^{-1},
\end{aligned}
$$

where $K=\left\{A_{1}, \ldots, A_{m}\right\}, L=\left\{B_{1}, \ldots, B_{n}\right\}$,

$$
\begin{aligned}
& A=i_{r, \beta}\left(A_{1} \wedge \ldots \wedge A_{m}\right), \\
& B=i_{r, \beta}\left(B_{1} \wedge \ldots \wedge B_{n}\right) .
\end{aligned}
$$

For $f^{a}(B)=0$, i.e., for $B=1$, we have that $i_{r, \beta}\left(B_{k}\right)=$ 1 for all $k \in\{1,2, \ldots, n\}$. E.

This means that $B_{k} \in E$ for $k \in\{1,2, \ldots, n\}$, that is, $L \subseteq$

Thus,

$$
\begin{aligned}
& \varphi\left(L\left[t^{\prime}, t^{\prime \prime}\right]\right) \\
= & \min \left\{\varphi\left(B_{1}\left[t^{\prime}, t^{\prime \prime}\right]\right), \ldots, \varphi\left(B_{n}\left[t^{\prime}, t^{\prime \prime}\right]\right)\right\} \\
= & \min \{1,1, \ldots, 1\}=1 \\
\geq & \min \left\{\theta_{2}, \varphi\left(K\left[t^{\prime}, t^{\prime \prime}\right]\right)\right\},
\end{aligned}
$$

where $\theta_{2}$ is the strength of the dependency $K{\stackrel{\theta_{2}}{\longrightarrow}}_{F} L$.

The obtained inequality means that the dependency is satisfied by $r^{\prime}$.

On the other hand, for $f^{a}(B)>0$,

$$
A<\frac{f^{a}\left(\frac{1}{2}\right)}{f^{a}(B)} .
$$

Note that it is either $A \leq \frac{1}{2}$ or $A>\frac{1}{2}$.

The assumption $A \leq \frac{1}{2}$ yields that at least one $A_{k} \in K, k$ $\in\{1,2, \ldots, m\}$ is such that $i_{r, \beta}\left(A_{k}\right) \leq \frac{1}{2}$, i.e., $A_{k} \notin E$.

Hence, $\varphi\left(A_{k}\left[t^{\prime}, t^{\prime \prime}\right]\right)=\theta^{\prime \prime}$, where $0 \leq \theta^{\prime \prime}<\theta^{\prime}$ for $\theta^{\prime}$ the smallest strength between strengths appearing in $C$ and $c$.

Thus, $\varphi\left(K\left[t^{\prime}, t^{\prime \prime}\right]\right)=\theta^{\prime \prime}$.

In other words,

$$
\begin{aligned}
& \varphi\left(L\left[t^{\prime}, t^{\prime \prime}\right]\right) \\
\geq & \theta^{\prime \prime}=\min \left\{\theta_{2}, \theta^{\prime \prime}\right\} \\
= & \min \left\{\theta_{2}, \varphi\left(K\left[t^{\prime}, t^{\prime \prime}\right]\right)\right\} .
\end{aligned}
$$

The assumption $A>\frac{1}{2}$, together with the relation (4) states that the quotient $\frac{f^{a}\left(\frac{1}{2}\right)}{f^{a}(B)}$ must be larger than 1 .

Hence, $B>\frac{1}{2}$.

Reasoning as above, we conclude that $L \subseteq E$, so $\varphi\left(L\left[t^{\prime}, t^{\prime \prime}\right]\right)=1$.
Thus, independently form $\theta_{2}$ and $\varphi\left(K\left[t^{\prime}, t^{\prime \prime}\right]\right)$, the inequality

$$
\varphi\left(L\left[t^{\prime}, t^{\prime \prime}\right]\right) \geq \min \left\{\theta_{2}, \varphi\left(K\left[t^{\prime}, t^{\prime \prime}\right]\right)\right\}
$$

is satisfied.

The given dependency is always satisfied by $r^{\prime}$.

Furthermore, in the multivalued case, the negated claim gives us

$$
\begin{aligned}
& \frac{1}{2}<i_{r, \beta}(\mathcal{K}) \\
&=i_{r, \beta}\left(\left(A_{1} \wedge \ldots \wedge A_{m}\right) \Rightarrow\right. \\
&\left.\left(\left(B_{1} \wedge \ldots \wedge B_{n}\right) \vee\left(C_{1} \wedge \ldots \wedge C_{p}\right)\right)\right) \\
&= \log _{a}\left(1+(a-1)^{1-A}\left(a^{B}-1\right)^{A}\right) \\
&=\left(f^{a}\left(A\left(f^{a}(B)\right)\right)\right)^{-1},
\end{aligned}
$$

where $K=\left\{A_{1}, \ldots, A_{m}\right\}, L=\left\{B_{1}, \ldots, B_{n}\right\}, M=U \backslash$ $(K \cup L)=\left\{C_{1}, \ldots, C_{p}\right\}$,

$$
\begin{aligned}
A= & i_{r, \beta}\left(A_{1} \wedge \ldots \wedge A_{m}\right), \\
B= & i_{r, \beta}\left(\left(B_{1} \wedge \ldots \wedge B_{n}\right) \vee\right. \\
& \left.\left(C_{1} \wedge \ldots \wedge C_{p}\right)\right) \\
= & \max \left\{i_{r, \beta}\left(B_{1} \wedge \ldots \wedge B_{n}\right),\right. \\
& \left.i_{r, \beta}\left(C_{1} \wedge \ldots \wedge C_{p}\right)\right\} .
\end{aligned}
$$

As above, $A \leq \frac{1}{2}$ or $A>\frac{1}{2}$.

If $A \leq \frac{1}{2}$, then $\varphi\left(K\left[t^{\prime}, t^{\prime \prime}\right]\right)=\theta^{\prime \prime}$.

In this scenario, we find the element $t^{\prime \prime \prime} \in$ $\left\{t^{\prime}, t^{\prime \prime}\right\}$, such that $t^{\prime \prime \prime}=t^{\prime}$, for which

$$
\begin{aligned}
\varphi\left(K\left[t^{\prime \prime \prime}, t^{\prime}\right]\right) & \geq \min \left\{\theta_{2}, \varphi\left(K\left[t^{\prime}, t^{\prime \prime}\right]\right)\right\}, \\
\varphi\left(L\left[t^{\prime \prime \prime}, t^{\prime}\right]\right) & \geq \min \left\{\theta_{2}, \varphi\left(K\left[t^{\prime}, t^{\prime \prime}\right]\right)\right\}, \\
\varphi\left(M\left[t^{\prime \prime \prime}, t^{\prime \prime}\right]\right) & \geq \min \left\{\theta_{2}, \varphi\left(K\left[t^{\prime}, t^{\prime \prime}\right]\right)\right\} .
\end{aligned}
$$

If $A>\frac{1}{2}$, then $\varphi\left(K\left[t^{\prime}, t^{\prime \prime}\right]\right)=1$.

For $f^{a}(B)=0$, i.e., for $B=1$, we have that $i_{r, \beta}\left(B_{k}\right)$ $=1$ for all $k \in\{1,2, \ldots, n\}$, or $i_{r, \beta}\left(C_{k}\right)=1$ for all $k \in$ $\{1,2, \ldots, p\}$.

This means that $B_{k} \in E$ for $k \in\{1,2, \ldots, n\}$ or $C_{k} \in E$ for $k \in\{1,2, \ldots, p\}$, i.e., that $L \subseteq E$ or $M \subseteq E$.

As in the functional case, we find that $\varphi\left(L\left[t^{\prime}, t^{\prime \prime}\right]\right)=1$ or $\varphi\left(M\left[t^{\prime}, t^{\prime \prime}\right]\right)=1$.

In the first scenario, we find the element $t^{\prime \prime \prime} \in\left\{t^{\prime}, t^{\prime \prime}\right\}$, such that $t^{\prime \prime \prime}=t^{\prime \prime}$, for which 5 , remains valid. 
On the other side, in the second case, 5 is fulfilled for $t^{\prime \prime \prime}$ $=t^{\prime}$.

Now, for $f^{a}(B)>0$, the relation (4) remains valid.

The assumption $A>\frac{1}{2}$ combined with 4 implies that

$$
\frac{f^{a}\left(\frac{1}{2}\right)}{f^{a}(B)}>1 .
$$

It immediately follows that $B>\frac{1}{2}$.

Thus, it is either $\varphi\left(L\left[t^{\prime}, t^{\prime \prime}\right]\right)=1$ or $\varphi\left(M\left[t^{\prime}, t^{\prime \prime}\right]\right)=1$.

In the first scenario, 5 is satisfied for $t^{\prime \prime \prime}=t^{\prime \prime}$.

On the other side, the case $\varphi\left(M\left[t^{\prime}, t^{\prime \prime}\right]\right)=1$ yields that (5) is valid for $t^{\prime \prime \prime}=t^{\prime}$.

The discussion conducted above proves that the given dependency (multivalued case) is always satisfied by $r^{\prime}$.

In order to complete the proof of the negated assumption of theorem, it is enough to prove that $c$ (in any case, functional or multivalued) is satisfied by $r^{\prime}$.

The proof follows from a similar reasoning as above.

This completes the proof.

Now, we prove the opposite claim of the claim given by Theorem 2 .

Theorem 3. Suppose that the assumptions of Theorem 2 hold. Then, if the fuzzy formula associated to dependency $c$ is valid when fuzzy formulas attached to set $C$ are correct, then, the dependency $c$ is a logical consequence of the set $C$ with respect to arbitrary fuzzy relation instanes.

Proof. We shall prove the assertion of theorem for the implication $I_{g^{b}}(x, y)$.

As in the proof of previous theorem, we apply the contraposition law.

Suppose that the negated claim of theorem holds true.

This means that $C$ is satisfied by $r^{\prime}$, and $c$ is not, for some $r^{\prime}=\left\{t^{\prime}, t^{\prime \prime}\right\}$.

First, we prove that the set of those $A \in U$, for which $\varphi\left(A\left[t^{\prime}, t^{\prime \prime}\right]\right)=1$, is non-empty set, and is a proper subset of $U$

Assume contrary, in the sense that $\varphi\left(A\left[t^{\prime}, t^{\prime \prime}\right]\right)=\theta^{\prime \prime}$ for all $A \in U$.

In the case when $c$ is not satisfied by $r^{\prime}$ in the functional case, we have that $\varphi\left(Y\left[t^{\prime}, t^{\prime \prime}\right]\right)$ is strictly smaller than

$$
\begin{aligned}
& \min \left\{\theta_{1}, \varphi\left(X\left[t^{\prime}, t^{\prime \prime}\right]\right)\right\} \\
= & \min \left\{\theta_{1}, \theta^{\prime \prime}\right\}=\theta^{\prime \prime} .
\end{aligned}
$$

This is not possible, however.

If $c$ is violated by $r^{\prime}$ as a fuzzy multivalued dependency, then $\sqrt{5}$ is not valid for any element $t^{\prime \prime \prime}$ of $r^{\prime}$, where the sets $K, L$ and $M$ are substituted by the sets $X, Y$ and $Z=U$ $\backslash(X \cup Y)$, and the linguistic strength $\theta_{2}$ is replaced by the strength $\theta_{1}$ (we consider the case $X \rightarrow{\stackrel{\theta_{1}}{\longrightarrow}}_{F} Y$ ).
In this, adapted form of $(5)$, it is clear that for $t^{\prime \prime \prime}=t^{\prime}$, the relations

$$
\begin{aligned}
& \varphi\left(X\left[t^{\prime \prime \prime}, t^{\prime}\right]\right) \geq \min \left\{\theta_{1}, \varphi\left(X\left[t^{\prime}, t^{\prime \prime}\right]\right)\right\}, \\
& \varphi\left(Y\left[t^{\prime \prime \prime}, t^{\prime}\right]\right) \geq \min \left\{\theta_{1}, \varphi\left(X\left[t^{\prime}, t^{\prime \prime}\right]\right)\right\}
\end{aligned}
$$

are fulfilled.

Taking into account the fact that (5) does not hold, we obtain that $\varphi\left(Z\left[t^{\prime \prime \prime}, t^{\prime \prime}\right]\right)$ is strictly smaller that $\theta^{\prime \prime}$. This, however, is not possible since $\varphi\left(Z\left[t^{\prime \prime \prime}, t^{\prime \prime}\right]\right)$ is equal to $\theta^{\prime \prime}$.

Summarizing what we just discussed, we come to the conclusion that the set of those $A \in U$, for which $\varphi\left(A\left[t^{\prime}, t^{\prime \prime \prime}\right]\right)$ $=1$, is a non-empty set.

Second, suppose contrary, in the sense that the set is equal to $U$.

Hence, if $c$ is violated by $r^{\prime}$, then $\varphi\left(Y\left[t^{\prime}, t^{\prime \prime}\right]\right)$ is strictly smaller than the minimum

$$
\min \left\{\theta_{1}, \varphi\left(X\left[t^{\prime}, t^{\prime \prime}\right]\right)\right\}
$$

which is not possible since $\varphi\left(Y\left[t^{\prime}, t^{\prime \prime}\right]\right)=1$, and

$$
\min \left\{\theta_{1}, \varphi\left(X\left[t^{\prime}, t^{\prime \prime}\right]\right)\right\} \leq 1
$$

In the multivalued case, the third inequality in (5) does not hold for $K=X, L=Y, M=Z, t^{\prime \prime \prime}=t^{\prime}$ and $\theta_{2}=\theta$.

Thus, $\varphi\left(Z\left[t^{\prime \prime \prime}, t^{\prime \prime}\right]\right)$, which is equal to 1 , is strictly smaller than the minimum $\min \left\{\theta_{1}, \varphi\left(X\left[t^{\prime}, t^{\prime \prime}\right]\right)\right\}$.

As we already seen, this is not possible,

We conclude, the set $E$ of the elements $A \in U$, such that $\varphi\left(A\left[t^{\prime}, t^{\prime \prime}\right]\right)=1$, is a non-empty set, and is a proper subset of $U$.

Determining the set $E$, we come to position to use the instance $r^{\prime}$ and the value $1 \in[0,1]$, to define a valuation $i_{r^{\prime}, 1}$, which is satisfied (larger than $\frac{1}{2}$ ), respectively falsified (smaller or equal to $\frac{1}{2}$ ), on the elements of $E$, respectively, on the elements of the complement of $E$.

Actually, we may assign some value $>\frac{1}{2}$ to the element of $E$, and some value $\leq \frac{1}{2}$ to the element of $U \backslash E$.

The proof of theorem will be completed if we prove that $C^{\prime}$ is satisfied by $i_{r^{\prime}, 1}$, while $c^{\prime}$ is not.

Suppose contrary, that the functional $\mathcal{K} \in C^{\prime}$ is violated by $i_{r^{\prime}, 1}$.

We obtain, 


$$
\begin{aligned}
& \frac{1}{2} \geq i_{r^{\prime}, 1}(\mathcal{K}) \\
& =i_{r^{\prime}, 1}\left(\left(A_{1} \wedge \ldots \wedge A_{m}\right) \Rightarrow\right. \\
& \left.\left(B_{1} \wedge \ldots \wedge B_{n}\right)\right)
\end{aligned}
$$

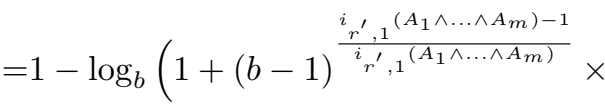

$$
\begin{aligned}
& \left.\times\left(b^{1-i_{r^{\prime}, 1}\left(B_{1} \wedge \ldots \wedge B_{n}\right)}-1\right)^{\frac{1}{{ }^{i} r^{\prime}, 1}\left(A_{1} \wedge \ldots \wedge A_{m}\right)}\right) \\
& =1-\log _{b}\left(1+(b-1)^{\frac{A-1}{A}}\left(b^{1-B}-1\right)^{\frac{1}{A}}\right) \text {, }
\end{aligned}
$$

where

$$
\begin{gathered}
A=i_{r^{\prime}, 1}\left(A_{1} \wedge \ldots \wedge A_{m}\right), \\
B=i_{r^{\prime}, 1}\left(B_{1} \wedge \ldots \wedge B_{n}\right),
\end{gathered}
$$

and $K=\left\{A_{1}, \ldots, A_{m}\right\}, L=\left\{B_{1}, \ldots B_{n}\right\}$, with the strength $\theta_{2}$.

As earlier, it follows that

$$
\frac{1}{2} \geq\left(g^{b}\left(\frac{1}{A} g^{b}(B)\right)\right)^{-1}
$$

For the sake of simplicity, we may assume that $g^{b}(B) \in$ $(0,+\infty)$.

The case $A=0$ together with $(6)$, yields that $g^{b}\left(\frac{1}{2}\right)=$ $+\infty$, which is not true having in mind that $g^{b}:[0,1] \rightarrow$ $[0,+\infty]$ is a strictly increasing function.

For $A \leq \frac{1}{2}$, the relation $\sqrt{6}$ gives us $\frac{g^{b}(B)}{g^{b}\left(\frac{1}{2}\right)}=0$.

This is not true.

Let $A>\frac{1}{2}$.

By $(6), g^{b}\left(\frac{1}{2}\right)>g^{b}(B)$.

Hence, $\varphi\left(L\left[t^{\prime}, t^{\prime \prime}\right]\right)=\theta^{\prime \prime}$.

Since $C$ is satisfied by $r^{\prime}$, we know that $\varphi\left(L\left[t^{\prime}, t^{\prime \prime}\right]\right)$ is larger or equal to the minimum

$$
\min \left\{\theta_{2}, \varphi\left(K\left[t^{\prime}, t^{\prime \prime}\right]\right)\right\}
$$

However, $\varphi\left(L\left[t^{\prime}, t^{\prime \prime}\right]\right)=\theta^{\prime \prime}$, while the minimum is equal to $\theta_{2}$ provided that $\varphi\left(K\left[t^{\prime}, t^{\prime \prime}\right]\right)=1$.

In any case, our selection of the strength $\theta^{\prime \prime}$ implies that the inequality $\theta^{\prime \prime} \geq \theta_{2}$ cannot be fulfilled.

Thus, a contradiction.

In other words, functional elements of $C^{\prime}$ are satisfied by $r^{\prime}$.

Secondly, suppose contrary, that the multivalued $\mathcal{K} \in C^{\prime}$ is violated by $i_{r^{\prime}, 1}$.

We obtain,

$$
\begin{aligned}
& 1 \geq 1-\log _{b}\left(1+(b-1)^{\frac{{ }_{i}{ }_{r^{\prime}, 1}\left(A_{1} \wedge \ldots \wedge A_{m}\right)-1}{i_{r^{\prime}, 1}\left(A_{1} \wedge \ldots \wedge A_{m}\right)}} \times\right. \\
& \left(b^{1-\max \left\{i_{r^{\prime}, 1}\left(B_{1} \wedge \ldots \wedge B_{n}\right), i_{r^{\prime}, 1}\left(C_{1} \wedge \ldots \wedge C_{p}\right)\right\}}-1\right) \\
& \left.\frac{1}{{ }_{i_{r^{\prime}, 1}\left(A_{1} \wedge \ldots \wedge A m\right)}}\right) \\
& =1-\log _{b}\left(1+(b-1)^{\frac{A-1}{A}}\left(b^{1-B}-1\right)^{\frac{1}{A}}\right) \\
& =\left(g^{b}\left(\frac{1}{A}\left(g^{b}(B)\right)\right)\right)^{-1},
\end{aligned}
$$

where

$$
\begin{aligned}
A= & i_{r^{\prime}, 1}\left(A_{1} \wedge \ldots \wedge A_{m}\right), \\
B= & \max \left\{i_{r^{\prime}, 1}\left(B_{1} \wedge \ldots \wedge B_{n}\right),\right. \\
& \left.i_{r^{\prime}, 1}\left(C_{1} \wedge \ldots \wedge C_{p}\right)\right\},
\end{aligned}
$$

and $K=\left\{A_{1}, \ldots, A_{m}\right\}, L=\left\{B_{1}, \ldots, B_{n}\right\}, M=U \backslash$ $(K \cup L)$, with the strength $\theta_{2}$.

As in the functional case, we assume that $g^{b}(B) \in$ $(0,+\infty)$.

The case $A=0$ leads to contradiction $g^{b}\left(\frac{1}{2}\right)=+\infty$.

The case $A \leq \frac{1}{2}$, gives the contradiction $\frac{g^{b}(B)}{g^{b}\left(\frac{1}{2}\right)}=0$.

Consider the case $A>\frac{1}{2}$.

As above, it follows that $\varphi\left(K\left[t^{\prime}, t^{\prime \prime}\right]\right)=1$.

Since $1 \geq\left(g^{b}\left(\frac{1}{A} g^{b}(B)\right)\right)^{-1}$, we arrive at $B<\frac{1}{2}$, i.e., we end up with $\varphi\left(L\left[t^{\prime}, t^{\prime \prime}\right]\right)=\theta^{\prime \prime}$ and $\varphi\left(M\left[t^{\prime}, t^{\prime \prime}\right]\right)=\theta^{\prime \prime}$.

Note that

$$
\begin{aligned}
& \min \left\{\theta_{2}, \varphi\left(K\left[t^{\prime}, t^{\prime \prime}\right]\right)\right\} \\
= & \min \left\{\theta_{2}, 1\right\}=\theta_{2} .
\end{aligned}
$$

Therefore, the last inequality in (5) does not hold for $t^{\prime \prime \prime}$ $=t^{\prime}$, and the second inequality in 5 is not true for $t^{\prime \prime \prime}=t^{\prime \prime}$.

Bearing in mind that $C$ is satisfied by $r^{\prime}$, we obtain a contradiction.

Thus, all multivalued elements of $C^{\prime}$ are satisfied by $i_{r^{\prime}, 1}$.

The proof of the fact that $c^{\prime}$ is violated by $i_{r^{\prime}, 1}$ is discussed similarly.

This completes the proof.

\section{APPLICATIONS AND EXAMPLES}

The results derived in this research may be applied to produce new fuzzy functional and fuzzy multivalued dependencies, not only by the application of the classical associated rules, but also from the resolution rules interpreted as the competent inference rules.

Example 1. The key role in this example will be played by the attributes $A, B, C$ and $D$ on given scheme $R$. The set $C_{1}$ of fuzzy functional and fuzzy multivalued dependencies on $R$ will consist of the following elements: $\{A\} \rightarrow{\stackrel{\theta_{1}}{\longrightarrow}}_{F}$ 
$\{B\},\{A, B\}{\stackrel{\theta_{2}}{\longrightarrow}}_{F}\{B, C\}$, and $\{D\} \rightarrow{\stackrel{\theta_{3}}{\longrightarrow}}_{F}\{B, C\}$. We shall show that the fuzzy functional dependency $c:\{D\}$ $\stackrel{\theta}{\rightarrow}_{F}\{C\}$ is a logical consequence of the set $C_{1}$, with $\theta=$ $\min \left\{\theta_{1}, \theta_{2}, \theta_{3}\right\}$.

Proof. I. Let's take a closer look at the dependencies: $\{A\}$ $\rightarrow{\stackrel{\theta_{1}}{\longrightarrow}}_{F}\{B\}$ and $\{A, B\}{\stackrel{\theta_{2}}{\longrightarrow}}_{F}\{B, C\}$.

We may write these dependencies as $X \rightarrow{\stackrel{\theta_{1}}{\rightarrow}}_{F} Y$ and $X \cup Y{\stackrel{\theta_{2}}{\longrightarrow}}_{F} Z$, where $X=\{A\}, Y=B$ and $Z=\{B, C\}$.

Recall the mixed pseudo-transitivity rule (see, [2, p. 173]): $X \rightarrow{\stackrel{\theta_{1}}{\longrightarrow}}_{F} Y$ and $X \cup Y{\stackrel{\theta_{2}}{\longrightarrow}}_{F} Z$ yield the dependency $X \stackrel{\theta^{\prime}}{\rightarrow}{ }_{F} Z \backslash Y$, where $\theta^{\prime}=\min \left\{\theta_{1}, \theta_{2}\right\}$.

In our case, it follow that $\{A\} \stackrel{\theta^{\prime}}{\rightarrow}\{C\}, \theta^{\prime}=$ $\min \left\{\theta_{1}, \theta_{2}\right\}$.

Now, we pay attention to dependencies: $\{D\} \rightarrow{\stackrel{\theta_{3}}{\longrightarrow}}_{F}$ $\{B, C\}$ and $\{A\}{\stackrel{\theta^{\prime}}{\rightarrow}}_{F}\{C\}$.

We recall the coalescence rule for fuzzy functional and fuzzy multivalued dependencies:

$X \rightarrow{\stackrel{\theta_{1}}{\longrightarrow}}_{F} Y$, and $Z \subseteq Y, W \cap Y=\emptyset$, together with $W{\stackrel{\theta_{2}}{\longrightarrow}}_{F}$ $Z$, implies that $X \stackrel{\min \left\{\theta_{1}, \theta_{2}\right\}}{\longrightarrow}{ }_{F} Z$.

We may write our dependencies in the form $X \rightarrow{\stackrel{\theta_{3}}{\longrightarrow}}_{F} Y$ and $W{\stackrel{\theta^{\prime}}{\rightarrow}}_{F} Z$, where $X=\{D\}, Y=\{B, C\}, Z=\{C\}$, and $W=\{A\}$.

Clearly, $Z \subseteq Y$ and $W \cap Y=\emptyset$.

Now, by the coalescence rule, the dependency $X$ $\stackrel{\min \left\{\theta_{3}, \theta^{\prime}\right\}}{\longrightarrow} F$ follows, i.e., $\{D\} \stackrel{\min \left\{\theta_{3}, \theta^{\prime}\right\}}{\longrightarrow} F\{C\}$ is true.

Proof. II. We can make the use of theorems derived above.

Namely, the obtained results give us the opportunity to replace the classical rules by the resolution rules, treating them as legitimate inference rules.

We have: $c_{1}^{\prime}: A \Rightarrow(B \vee(C \wedge D)), c_{2}^{\prime}:(A \wedge B) \Rightarrow$ $(C \wedge D), c_{3}^{\prime}: D \Rightarrow((B \wedge C) \vee A), c^{\prime}: D \Rightarrow C$.

Since $(p \Rightarrow q) \equiv(\neg p \vee q)$, we may write:

$$
\begin{aligned}
c_{1}^{\prime} & \equiv A \Rightarrow(B \vee(C \wedge D)) \\
& \equiv \neg A \vee(B \vee(C \wedge D)) \\
& \equiv \neg A \vee((B \vee C) \wedge(B \vee D)) \\
& \equiv(\neg A \vee B \vee C) \wedge(\neg A \vee B \vee D) .
\end{aligned}
$$

Similarly, $c_{2}^{\prime}: \neg A \wedge(\neg A \vee \neg B \vee C)$, $c_{3}^{\prime}:(A \vee B \vee \neg D) \wedge(A \vee C \vee \neg D), \neg c^{\prime}: \neg C \wedge D$

Our goal is to prove that the fuzzy formula $c^{\prime}: C \vee \neg D$ is correct.

Taking into account the fact that the formulas $c_{1}^{\prime}, c_{2}^{\prime}$ and $c_{3}^{\prime}$ are already true, it is enough to show that the formula $c_{1}^{\prime} \wedge$ $c_{2}^{\prime} \wedge c_{3}^{\prime} \wedge \neg c^{\prime}$ cannot stand alone.

Let $F^{*}$ be the set of all conjunctive terms of the formulas $c_{1}^{\prime}, c_{2}^{\prime}, c_{3}^{\prime}$ and $\neg c^{\prime}$.
Thus,

$$
\begin{aligned}
F^{*}= & \{\neg A \vee B \vee C, \neg A \vee B \vee D, \neg A, \\
& A \vee B \vee \neg D, A \vee C \vee \neg D, \neg C, D\} .
\end{aligned}
$$

We obtain the following resolvents: $A \vee C \vee \neg D$ and $D$ gives $A \vee C$. Then $A \vee C$ and $\neg C$ gives $A$. Since $\neg A$ is already given, $A$ and $\neg A$ yield a contradiction.

Hence, $\neg c^{\prime}$ is not correct, so $c^{\prime}$ is valid.

\section{CONCLUSION}

In this paper we consider and prove several equivalences between some of the elements of similarity-based databases (fuzzy functional and fuzzy multivalued dependencies), and some of the elements of fuzzy logic theory on the other side (fuzzy formulas).

The requirement that some fuzzy multivalued dependency is actively satisfied by given two-element fuzzy relation instance is reduced to equivalent condition that the attached fuzzy formula is valid under appropriate valuation, keeping particular database condition that the tuples of the instance are mutually conformant (up to some extent) on the leading set of attributes (Theorem 11).

The equivalence is extended to sets of fuzzy dependencies and fuzzy formulas in the form of Theorems 2 and 3

It has been proven that some set of fuzzy dependencies yields some particular fuzzy dependency (in both, the world of two-element and the world of arbitrary fuzzy relation instances) if and only if the correctness of the associated set of fuzzy formulas yields the validity of the particular fuzzy formula.

The results are derived assuming that the attributes in the universal set of attributes are turned into fuzzy formulas with respect to introduced valuations (depending on two-element instances and values in the unit interval $[0,1]$ ). Generalizing described concept to sets of attributes, fuzzy dependencies are also treated as fuzzy formulas.

The application of the results is presented by the example discussed above, where is shown that steps in the classical proofs (of the fact that some dependency follows from a set of dependencies), can be fully automated.

The research can be transferred to more general settings of vague functional and vague multivalued dependencies.

\section{FINAL REMARKS}

In order to demonstrate the impact of this research to electrical and computer engineering as well as to prove its practical applicability, we consider the following example.

Let "Name", "Intelligence", "Success", and "Salary" be some attributes on given scheme $R$.

We put $U$ to be the universal set of attributes, i.e.,

$$
U=\{\text { Name, Intelligence, Success, Salary }\} \text {. }
$$

Suppose that $N, I, S$ and $S^{\prime}$ are the domains of the at- 
tributes listed above, and that they are introduced as follows.

$$
\begin{aligned}
N & =\{\text { Neo, Tom }, \text { Koyuki }\}, \\
I & =\{100,120,145\} \\
S & =\{\text { low, high }\} \\
S^{\prime} & =\{\text { high, very }- \text { high }\} .
\end{aligned}
$$

First, we select the similarity relations on $N, I, S$ and $S^{\prime}$. In order to be in line with the introduced notation, we require these relations to be reflexive, symmetric and max-min transitive.

Thus, we introduce $s_{1}: S \times S \rightarrow[0,1]:$ by $s_{1}(N e o, N e o)$ $=s_{1}($ Tom, Tom $)=$

$s_{1}($ Koyuki, Koyuki $)=1$, and $s_{1}($ Neo, Tom $)$

$=s_{1}($ Tom, Neo $)=0.9, s_{1}($ Neo, Koyuki $)=$

$s_{1}($ Koyuki, Neo $)=0.9, s_{1}($ Tom, Koyuki $)=$

$s_{1}($ Koyuki, Tom $)=0.9$.

In short, we require that $s_{1}(a, a)=1$ and $s_{1}(a, b)=0.9$.

Since $s_{1}$ is clearly reflexive and transitive, we have to prove that $s_{1}$ is also max-min transitive.

In other words, we have to prove that the condition

$$
\begin{aligned}
& s(a, c) \\
\geq & \max _{b \in A}\{\min \{s(a, b), s(b, c)\}\}
\end{aligned}
$$

is valid for $s=s_{1}, A=N$ and all $a, c \in N$.

For example,

$$
\begin{aligned}
& \max _{b \in N}\left\{\min \left\{s_{1}(\text { Tom }, b), s_{1}(\text { b Koyuki })\right\}\right\} \\
= & \max \left\{\operatorname { m i n } \left\{s_{1}(\text { Tom }, \text { Neo }),\right.\right. \\
& \left.s_{1}(\text { Neo }, \text { Koyuki })\right\}, \\
& \min \left\{s_{1}(\text { Tom }, \text { Tom }),\right. \\
\left.s_{1}(\text { Tom }, \text { Koyuki })\right\}, & \min \left\{s_{1}(\text { Tom }, \text { Koyuki }),\right. \\
& \left.\left.s_{1}(\text { Koyuki, Koyuki })\right\}\right\} \\
= & \max \{\min \{0.9,0.9\}, \min \{1,0.9\}, \\
& \min \{0.9,1\}\} \\
= & \max \{0.9,0.9,0.9\} \\
= & 0.9=s_{1}(\text { Tom }, \text { Koyuki }) .
\end{aligned}
$$

Thus, (7) holds true for $a=$ "Tom", $c=$ "Koyuki".

Note that in the case $a \neq c$, the minimum on the right hand side of (7) is 0.9 for all $b \in N$.

In particular, it can happen that $a \neq b \neq c$ or that $a=b \neq$ $c$ or $a \neq b=c$.

In any case, $s_{1}(a, b)$ or $s_{1}(b, c)$ is 0.9 .

Hence, in any case, the minimum is always 0.9 .
Since the left hand side of (7) in such scenario is also 0.9, the condition (7) follows.

Note that for $a=c$, the left hand side in (7) is 1 , so the condition is automatically satisfied, provided that the right hand side is not larger than 1 .

So, $s_{1}$ is a similarity relation on $N$.

Let $s_{2}: I \times I \rightarrow[0,1]$ be determined by the equations $s_{2}(100,100)=s_{2}(120,120)=s_{2}(145,145)=1$, and $s_{2}(100,120)=s_{2}(120,100)=0.8$,

$s_{2}(100,145)=s_{2}(145,100)=0.8, s_{2}(120,145)=$ $s_{2}(145,120)=0.8$.

Reasoning in the same way as in the case of $s_{1}$, we conclude that $s_{2}$ is a similarity relation on $I$.

Put $s_{3}: S \times S \rightarrow[0,1]$ to be the relation defined by: $s_{3}($ low, low $)=s_{3}($ high, high $)=1$, and $s_{3}($ low, high $)=$ $s_{3}($ high, low $)=0.7$.

We obtain,

$$
\begin{aligned}
& \max _{b \in S}\left\{\min \left\{s_{3}\left(\text { low }, \text { b) }, s_{3}(\text { b, high })\right\}\right\}\right. \\
= & \max \left\{\operatorname { m i n } \left\{s_{3}(\text { low }, \text { low }),\right.\right. \\
& \left.s_{3}(\text { low, high })\right\}, \\
& \min \left\{s_{3}(\text { low }, \text { high }),\right. \\
& \left.\left.s_{3}(\text { high, high })\right\}\right\} \\
= & \max \{\min \{1,0.7\}, \min \{0.7,1\}\} \\
= & \max \{0.7,0.7\} \\
= & 0.7=s_{3}(\text { low }, \text { high }) .
\end{aligned}
$$

Consequently, $s_{3}$ is a similarity relation on $S$.

Finally, we define $s_{4}: S^{\prime} \times S^{\prime} \rightarrow[0,1]$ by: $s_{4}($ high, high $)=s_{4}($ very - high, very - high $)=1$, $s_{4}($ high, very - high $)=s_{4}($ very - high, high $)=0.6$.

As in the case of the relation $s_{3}$, we immediately have that $s_{4}$ is a similarity relation on $S^{\prime}$.

Put $r=\left\{t_{1}, t_{2}\right\}$ to be the fuzzy relation instance on $R$ with tuples defined as follows.

We put the elements of $t_{1}$ to be: $\{$ Neo, Koyuki $\},\{120\}$, $\{$ low, high $\},\{$ high $\}$, and the elements of $t_{2}$ to be $\{$ Tom $\},\{100,120,145\},\{$ high $\}$, $\{$ high, very - high $\}$.

Let's find the similarities between tuples $t_{1}$ and $t_{2}$ on attributes in $U$, i.e., the conformances $\varphi\left(A\left[t_{1}, t_{2}\right]\right)$, $A \in U$ for the realistic scenario described by $r$.

We have,

$$
\begin{aligned}
& \varphi\left(\text { Name }\left[t_{1}, t_{2}\right]\right) \\
= & \min \left\{\operatorname { m i n } _ { x \in \{ \text { Neo,Koyuki } \} } \left\{\max _{y \in\{\text { Tom }\}}\{\right.\right. \\
& \left.\left.s_{1}(x, y)\right\}\right\}, \\
& \min _{x \in\{\text { Tom }\}}\left\{\max _{y \in\{\text { Neo }, \text { Koyuki }\}}\{\right. \\
& \left.\left.\left.s_{1}(x, y)\right\}\right\}\right\}
\end{aligned}
$$




$$
\begin{aligned}
& =\min \left\{\operatorname { m i n } \left\{s_{1}(\text { Neo,Tom }),\right.\right. \\
& \left.s_{1}(\text { Koyuki,Tom })\right\} \text {, } \\
& \max \left\{s_{1}(\text { Tom, Neo }),\right. \\
& \left.s_{1}(\text { Tom }, \text { Koyuki) }\}\right\} \\
& =\min \{\min \{0.9,0.9\}, \max \{0.9,0.9\}\} \\
& =\min \{0.9,0.9\}=0.9 \text {, } \\
& \varphi\left(\text { Intelligence }\left[t_{1}, t_{2}\right]\right) \\
& =\min \left\{\operatorname { m i n } _ { x \in \{ 1 2 0 \} } \left\{\max _{y \in\{100,120,145\}}\{\right.\right. \\
& \left.\left.s_{2}(x, y)\right\}\right\} \text {, } \\
& \min _{x \in\{100,120,145\}}\left\{\max _{y \in\{120\}}\{\right. \\
& \left.\left.\left.s_{2}(x, y)\right\}\right\}\right\} \\
& =\min \left\{\operatorname { m a x } \left\{s_{2}(120,100)\right.\right. \text {, } \\
& \left.s_{2}(120,120), s_{2}(120,145)\right\} \text {, } \\
& \min \left\{s_{2}(100,120)\right. \text {, } \\
& \left.\left.s_{2}(120,120), s_{2}(145,120)\right\}\right\} \\
& =\min \{\max \{0.8,1,0.8\}, \min \{0.8,1,0.8\}\} \\
& =\min \{1,0.8\}=0.8 \text {, } \\
& \varphi\left(\text { Success }\left[t_{1}, t_{2}\right]\right) \\
& =\min \left\{\operatorname { m i n } _ { x \in \{ l o w , h i g h \} } \left\{\max _{y \in\{\text { high }\}}\{\right.\right. \\
& \left.\left.s_{3}(x, y)\right\}\right\} \\
& \min _{x \in\{\text { high }\}}\left\{\max _{y \in\{\text { low,high }\}}\{\right. \\
& \left.\left.\left.s_{3}(x, y)\right\}\right\}\right\} \\
& =\min \left\{\operatorname { m i n } \left\{s_{3}(\text { low, high })\right.\right. \text {, } \\
& \left.s_{3}(\text { high }, \text { high })\right\} \text {, } \\
& \max \left\{s_{3}(\text { high, low }),\right. \\
& \left.s_{3}(\text { high, high) }\}\right\} \\
& =\min \{\min \{0.7,1\}, \max \{0.7,1\}\} \\
& =\min \{0.7,1\}=0.7 \text {, } \\
& \varphi\left(\text { Salary }\left[t_{1}, t_{2}\right]\right) \\
& =\min \left\{\operatorname { m i n } _ { x \in \{ h i g h \} } \left\{\max _{y \in\{h i g h, v e r y-h i g h\}}\{\right.\right. \\
& \left.\left.s_{4}(x, y)\right\}\right\} \text {, } \\
& \min _{x \in\{\text { high,very-high }\}}\left\{\max _{y \in\{\text { high }\}}\{\right. \\
& \left.\left.\left.s_{4}(x, y)\right\}\right\}\right\}
\end{aligned}
$$

$$
\begin{aligned}
= & \min \left\{\operatorname { m a x } \left\{s_{4}(\text { high }, \text { high }),\right.\right. \\
& \left.s_{4}(\text { high, very }- \text { high })\right\}, \\
& \min \left\{s_{4}(\text { high, high }),\right. \\
& \left.\left.s_{4}(\text { very }- \text { high, high })\right\}\right\} \\
= & \min \{\max \{1,0.6\}, \min \{1,0.6\}\} \\
= & \min \{1,0.6\}=0.6 .
\end{aligned}
$$

Consider the following fuzzy functional and fuzzy multivalued dependencies on $R:\{$ Name $\}$ $\rightarrow \stackrel{0.79}{\longrightarrow}_{F}\{$ Intelligence $\},\{$ Name, Intelligence $\}$ $\stackrel{0.6}{\longrightarrow}_{F}\{$ Intelligence, Success $\},\{$ Salary $\}$ $\rightarrow \stackrel{0.98}{\longrightarrow}_{F}\{$ Intelligence, Success $\},\{$ Salary $\}$ $\stackrel{0.6}{\longrightarrow}_{F}\{$ Success $\}$.

It is not so hard to see that $r$ satisfies these dependencies.

Namely, for tuples $t_{1}, t_{2} \in r$, there is the tuple $t_{2} \in r$, such that

$$
\begin{aligned}
& \varphi\left(\text { Name }\left[t_{2}, t_{1}\right]\right) \\
= & 0.9>0.79=\min \{0.79,0.9\} \\
= & \min \left\{0.79, \varphi\left(\text { Name }\left[t_{1}, t_{2}\right]\right)\right\}, \\
& \varphi\left(\text { Intelligence }\left[t_{2}, t_{1}\right]\right) \\
= & 0.8>0.79=\min \left\{0.79, \varphi\left(\text { Name }\left[t_{1}, t_{2}\right]\right)\right\}, \\
& \varphi\left(\left\{\text { Success, Salary }\left[t_{2}, t_{2}\right]\right)\right. \\
= & 1 \geq \min \left\{0.79, \varphi\left(\text { Name }\left[t_{1}, t_{2}\right]\right)\right\} .
\end{aligned}
$$

Furthermore,

$$
\begin{aligned}
& \varphi\left(\{\text { Name }, \text { Intelligence }\}\left[t_{1}, t_{2}\right]\right) \\
= & \min \left\{\varphi\left(\text { Name }\left[t_{1}, t_{2}\right]\right),\right. \\
& \left.\varphi\left(\text { Intelligence }\left[t_{1}, t_{2}\right]\right)\right\} \\
= & \min \{0.9,0.8\}=0.8, \\
& \varphi\left(\{\text { Intelligence }, \text { Success }\}\left[t_{1}, t_{2}\right]\right) \\
= & \min \left\{\varphi\left(\text { Intelligence }\left[t_{1}, t_{2}\right]\right),\right. \\
& \left.\varphi\left(\text { Success }\left[t_{1}, t_{2}\right]\right)\right\} \\
= & \min \{0.8,0.7\}=0.7,
\end{aligned}
$$

so

$$
\begin{aligned}
& \varphi\left(\{\text { Intelligence, Success }\}\left[t_{1}, t_{2}\right]\right) \\
= & 0.7>0.6=\min \{0.6,0.8\} \\
= & \min \left\{0.6, \varphi\left(\{\text { Name }, \text { Intelligence }\}\left[t_{1}, t_{2}\right]\right)\right\} .
\end{aligned}
$$

We also know that for tuples $t_{1}, t_{2} \in r$, there exists the tuple $t_{2} \in r$, such that 


$$
\begin{aligned}
& \varphi\left(\text { Salary }\left[t_{2}, t_{1}\right]\right) \\
= & 0.6=\min \{0.98,0.6\} \\
= & \min \left\{0.98, \varphi\left(\text { Salary }\left[t_{1}, t_{2}\right]\right)\right\}, \\
& \varphi\left(\{\text { Intelligence, Success }\}\left[t_{2}, t_{1}\right]\right) \\
= & 0.7>0.6=\min \left\{0.98, \varphi\left(\text { Salary }\left[t_{1}, t_{2}\right]\right)\right\}, \\
& \varphi\left(\text { Name }\left[t_{2}, t_{2}\right]\right) \\
= & 1 \geq \min \left\{0.98, \varphi\left(\text { Salary }\left[t_{1}, t_{2}\right]\right)\right\} .
\end{aligned}
$$

Finally,

$$
\begin{aligned}
& \varphi\left(\text { Success }\left[t_{1}, t_{2}\right]\right) \\
= & 0.7>0.6=\min \{0.6,0.6\} \\
= & \min \left\{0.6, \varphi\left(\text { Success }\left[t_{1}, t_{2}\right]\right)\right\} .
\end{aligned}
$$

Thus, the dependencies listed above are all satisfied by $r$. Regarding the obtained results, we point out the following remarks.

First, let $C$ denote the set of the dependencies:

$\{$ Name $\} \rightarrow \stackrel{0.79}{\longrightarrow}$ F Intelligence $\},$

$\{$ Name, Intelligence $\} \stackrel{0.6}{\longrightarrow}_{F}$

$\{$ Intelligence, Success $\},\{$ Salary $\} \rightarrow \stackrel{0.98}{\longrightarrow}_{F}$

$\{$ Intelligence, Success $\}$.

Denote by $c$ the dependency $\{$ Salary $\} \stackrel{0.6}{\longrightarrow}_{F}\{$ Success $\}$.

The obtained conclusions could be treated in the way that $r$ satisfies $c$ and each of the elements in $C$ independently of each other. However, we could wonder if or not the set $C$ implies $c$ in particular case of fuzzy relation instance $r$, or more generally, in the world of two-element fuzzy relation instances, or even more generally, in the world of arbitrary fuzzy relations.

Recall Example 1 (see, Section 4).

Putting $A, B, C, D$ in Example 1 to be "Name", "Intelligence", "Success", "Salary", and $\theta_{1}, \theta_{2}, \theta_{3}$ to be $0.79,0.6$, 0.98 , we find that the set of dependencies $C$ yields the dependency $c$ (in arbitrary fuzzy relations). This fact shows that the results derived within this discussion can be generalized, and what is more important, can be obtained in an automated way. Namely, the assertion of Example 1 is achieved via inference rules (proof I), as well as via resolution principle, i.e., via automatization (proof II). Thus, the obtained connection between conclusions of this section and the possibility of automatization (enabled by the current research in total), provides the impact that this research could achieve in electrical and computer engineering, showing also its practical applicability.

\section{REFERENCES}

[1] R. Fagin, "Multivalued dependencies and a new normal form for relational databases," ACM. Trans. Database Systems, vol. 2, pp. 262-278, 1977.

[2] M. Sozat and A. Yazici, "A complete axiomatization for fuzzy functional and multivalued dependencies in fuzzy database relations," Fuzzy Sets and Systems, vol. 117, pp. 161-181, 2001.
[3] A. Yazici, E. Gocmen, B. P. Buckles, R. George, and F. E. Petry, "An integrity constraint for a fuzzy relational database," in Proc. IEEE Int. Conf. Fuzzy Systems 1993, FUZZ-IEEE 93, pp. 496-499.

[4] R. C. Tripathy and P. C. Saxena, "Multivalued dependencies in fuzzy relational databases," Fuzzy Sets and Systems, vol. 38, pp. 267-279, 1990.

[5] M. Nakata, "Dependencies in fuzzy databases: multivalued dependency," in Proceedings of IEEE Int. Conf. on Fuzzy Systems, (FUZZ-IEEE 96), 1996, pp. 15911597.

[6] J.-D. Ullman, Principles of Database Systems. Rockville: Computer Science Press, 1982.

[7] C. Giardina, I. Sack, and D. Sinha, Fuzzy Field Relational Database Tech. Report 8332. Hoboken: Elect Engng. and Computer Science Dept., Stevens Institute of Technology, 1983.

[8] H. Prade, "The connection between Lipski's approach to incomplete information data bases and Zadeh's Possibility Theory," in Proc. Int. Conf. System Meth., 1982, pp. 402-408.

[9] H. Prade, "Lipski's approach to incomplete information databases restated and generalized in the setting of Zadeh's Possibility Theory,' Inform. Systems, vol. 9, pp. 27-42, 1984.

[10] H. Prade and C. Testemale, "Generalizing database relational algebra for the treatment of incomplete/uncertain informations and vague queries," in 2nd NAFIP Workshop, 1983.

[11] B. P. Buckles and F. E. Petry, "A fuzzy representation of data for relational databases," Fuzzy Sets and Systems, vol. 7, pp. 213-216, 1982.

[12] B. P. Buckles and F. E. Petry, "Fuzzy databases and their applications," Fuzzy Inform. Decision Process, vol. 2, pp. 361-371, 1982.

[13] B. P. Buckles and F. E. Petry, "Uncertainty models in information and database systems," J. Inform. Sci., vol. 11, pp. 77-87, 1985.

[14] N. Dukić, Dž. Gušić, A. Muratović-Ribić, A. Alihodžić, E. Tabak, and H. Dukić, "From fuzzy dependences to fuzzy formulas and vice versa, for Kleene-Dienes fuzzy implication operator," WSEAS Trans. on Systems and Control, vol. 13, pp. 285-297, 2018.

[15] R. C. T. Lee, "Fuzzy Logic and the Resolution Principle," J. Assoc. Comput. Mach., vol. 19, pp. 109-119, 1972.

[16] Dž. Gušić, "Continuous Maps in Fuzzy Relations," WSEAS Trans. on Systems and Control, vol. 13, pp. 324-344, 2018.

[17] Dž. Gušić, "Reichenbach and $f$-generated implications in fuzzy database relations," WSEAS Trans. on Systems and Control, vol. 14, pp. 71-89, 2019.

[18] Dž. Gušić, "Continuous Functions, g-generated Implications and Fuzzy Dependencies in Fuzzy Relation Instances," in 2018 International Conference on Applied Mathematics and Computational Science (ICAMCS.NET), Budapest, 2018, pp. 98-109.

[19] N. Dukić, Dž. Gušić, and N. Kajmović, "On Fuzzy Dependencies and $g$ - generated Fuzzy Implications in 
Fuzzy Relations," Int. J. of Circuits, Systems and Sygnal Processing, vol. 12, pp. 285-297, 2018.

[20] S. Nesimović and Dž. Gušić, "Klir-Yuan Fuzzy Implication in Fuzzy Relations," in 2019 International Conference on Control, Artificial Intelligence, Robotics and Optimization (ICCAIRO), Athens, 2019, pp. 163-169.

[21] Dž. Gušić, "Soundness and Completeness of Inference Rules for New Vague Functional Dependencies," in N. Mastorakis, V. Mladenov and A. Bulucea (eds.) MATEC Web of Conferences, Vol. 292, 23rd International Conference on Circuits, Systems, Communications and Computers (CSCC 2019), Athens, 2019, pp. $1-9$.

[22] Dž. Gušić, "Soundness of Inference Rules for New Vague Multivalued Dependencies," in

N. Mastorakis, V. Mladenov and A. Bulucea (eds.) MATEC Web of Conferences, Vol. 292, 23rd International Conference on Circuits, Systems, Communications and Computers (CSCC 2019), Athens, 2019, pp. $1-7$.

[23] A. Lu and W. Ng, "Managing Merged Data by Vague Functional Dependencies," in P. Atzeni, W. Chu, H. Lu, S. Zhou, T. W. Ling (eds.) ER 2004 LNCS, BerlinHeidelberg, 2004, pp. 259-272.

[24] S. M. Chen, "Similarity Measures Between Vague Sets and Betveen Elements," IEEE Transactions on Systems, Man and Cybernetics, vol. 27, pp. 153-159, 1997.

[25] D. H. Hong and C. Kim, "A note on Similarity Measures Between Vague Sets and Between Elements," Information Sciences, vol. 115, pp. 83-96, 1999.

[26] F. Li and Z. Xu, "Measures of Similarity Between Vague Sets," Journal of Software, vol. 12, pp. 922-927, 2001.

[27] E. Szmidt and J. Kacprzyk, "Distances Between Intuitionistic Fuzzy Sets," Fuzzy Sets and Systems, vol. 58, pp. 267-279, 1990.

[28] Dž. Gušić, Z. Šabanac, and S. Nesimović, "On Soundness of Various Inference Rules for Vague Functional Dependencies," in 3rd International

Conference on Control, Artificial Intelligence,

Robotics and Optimization (ICCAIRO), Athens, 2019, pp. 182-188.

[29] Dž. Gušić, “On Completeness of Inference Rules for Vague Functional and Vague Multivalued Dependencies in two-element Vague Relation Instances," WSEAS Trans. on Systems and Control, vol. 14, pp. 232-238, 2019.

[30] Dž. Gušić, "Completeness of Inference Rules for New Vague Multivalued Dependencies," WSEAS Trans. on Mathematics, vol. 18, pp. 295-306, 2019.

[31] Dž. Gušić, "Vague Functional Dependencies and Resolution Principle," WSEAS Trans. on Mathematics, vol. 18, pp. 288-294, 2019.

[32] Dž. Gušić, "Vague Multivalued Dependencies and Resolution Principle," WSEAS Trans. on Mathematics, vol. 18, pp. 257-263, 2019.

[33] N. Dukić, Dž. Gušić, and N. Kajmović, “On Equivalences Between Fuzzy Dependencies and Fuzzy Formulas Satisfiability for Yagers Fuzzy Implication Operator," WSEAS Trans. on Mathematics, vol. 17, pp. 35-
43, 2018.

[34] J. Mishra and S. Ghosh, "A Vague Multivalued Data Dependency," Fuzzy Inf. Eng., vol. 4, pp. 459-473, 2013.

[35] M. Baczyński and B. Jayaram, Fuzzy Implications. Berlin-Heidelberg: Springer-Verlag, 2008.

[36] Y. Shi, "A Deep Study of Fuzzy Implications," Ph.D. dissertation, Faculty of Science, Ghent Univ., Ghent, 2009.

Contribution of individual authors to the creation of a scientific article (ghostwriting policy)

Author Contributions: Please, indicate the role and the contribution of each author:

Dženan Gušić has conceptualized the research and organized the paper.

Sanela Nesimović derived the results, discussed their applications, and made examples and the literature review.

Follow: http://naun.org/main/format/contributor-role.pdf

Sources of funding for research presented in a scientific article or scientific article itself

Report potential sources of funding if there is any

\section{Creative Commons Attribution License 4.0 (Attribution 4.0 International, CC BY 4.0)}

This article is published under the terms of the Creative Commons Attribution License 4.0 https://creativecommons.org/licenses/by/4.0/deed.en_US 